Rev. SINAPSIS, Edición № 9, Vol. 2, Diciembre 2016

ISSN $1390-9770$

\title{
Los recursos didácticos y el aprendizaje significativo en los estudiantes de bachillerato'
}

\section{Recursos didácticos y el aprendizaje significativo}

\author{
Elvira Geoconda Villacreses Veliz M.Sc. ${ }^{(1)}$ \\ Adela del Jesús Lucio Pillasagua M.Sc. ${ }^{(2)}$ \\ Carlos Hernán Romero Yela Dr. \\ (1)Universidad Estatal del Sur de Manabí, Jipijapa, Ecuador \\ ${ }^{(2)}$ Universidad Estatal del Sur de Manabí, Jipijapa, Ecuador \\ ${ }^{(3)}$ Instituto Ecuatoriano de Seguridad Social, Jipijapa, Ecuador
}

Contacto: yoqui.rovi.2010@ hotmail.com

Receptado: 20/09/2016 Aceptado: 15/11/2016

\section{Resumen}

La presente investigación denominada "Los recursos didácticos y el aprendizaje significativo en el Colegio Nacional Alejo Lascano, de la ciudad de Jipijapa, provincia de Manabí”, tuvo como finalidad contribuir al fortalecimiento del sistema educativo. Con la presente investigación, se ha detectado que gran parte de los docentes, no han aplicado recursos didácticos y metodologías eficientes, no utilizan materiales didácticos creativos ni metodologías innovadoras y esto se refleja en el proceso enseñanza aprendizaje. Los recursos didácticos son los productos diseñados para ayudar en los procesos de enseñanza, como medios de expresión; y el aprendizaje significativo estimula la función de los sentidos para acceder de manera fácil la adquisición de conceptos, habilidades y destrezas. Para recopilar la información de la presente investigación, se hizo uso del método bibliográfico-documental, para optimizar y desarrollar el trabajo investigativo, se utilizó la investigación de campo; las técnicas utilizadas fueron la encuesta y la entrevista. Definido el nivel de calidad de la educación y con la recopilación de los resultados se elaboró la propuesta para potenciar el interés estudiantil y mejorar el rendimiento escolar a través de la aplicación de los recursos didácticos y metodologías activas que conlleven al aprendizaje significativo y mejorar el proceso educativo. 
Rev. SINAPSIS, Edición № 9, Vol. 2, Diciembre 2016

Palabras clave: Fortalecer, potenciar, enseñanza, métodos, técnicas

\title{
The Didactic Resources and Significant Learning in the students of bachelor
}

\begin{abstract}
This research called "The teaching and meaningful learning resources at the National College Alejo Lascano, City Jipijapa, Manabí province", aimed to help strengthen the educational system. With this research, we have found that most teachers have not applied teaching resources and efficient methodologies, which develops educational work with new active and participatory techniques according to the structure of each subject, it is not being used or developing creative teaching materials and innovative methodologies and this is reflected in the teaching-learning process. Teaching resources are products designed to aid in the learning process as an instrument or means of expression and / or communication and meaningful learning stimulates the senses function for easy access to the acquisition of concepts how, skills, attitudes or skills. To gather information from this investigation, use was made of bibliographic documentary method to optimize and develop the research work, field research was used; the techniques used were the survey and interview. Defined the quality of education in all its aspects and with the compilation of the results the proposal was developed to boost student interest and improve school performance through the application of teaching resources and active methodologies that lead to meaningful learning and improve the educational process at the National College Alejo Lascano.
\end{abstract}

Keywords: Strengthening, empowering, teaching methods, techniques 


\section{Introducción}

El problema de la educación ha sido durante toda la historia de la humanidad un tema polémico, candente, de amplio debate, articulado con las concepciones pedagógicas y filosóficas y a las condiciones sociales, económicas, políticas, ideológicas y culturales por la que ha atravesado y atraviesa la humanidad.

Se puntualiza que la educación se sigue practicando en la actualidad entre los alumnos y docentes con la comunicación manual, con lapiceros o bolígrafos y, de manera mecánica, a través de la producción seriada de informaciones, y son muy pocos los que utilizan y producen recursos informacionales digitales(Aparici, 2011)

En el proceso enseñanza aprendizaje, se ha detectado que gran parte de los docentes, muestran deficiencias en la aplicación de recursos didácticos y metodologías participativas con sus estudiantes que permitan el desarrollo de la labor educativa acorde con el momento histórico que se está viviendo, existe carencia en la elaboración y utilización de materiales didácticos creativos acompañados de metodologías innovadoras reflejándose esta situación en el aprendizaje de los estudiantes. Las dificultades expuestas orientan a formular el problema central de la investigación el cual está orientado hacia la adquisición del aprendizaje significativo.

Una buena enseñanza promueve un cambio en el aprendizaje significativo que busca una nueva información con estructura cognitiva de la persona que aprende.

Se ha señalado que la "enseñanza" se observa como pasa de ser considerada un proceso transmisionalista, en el que el contenido se encuentra sobre valorado; a ser un proceso promotor y dinamizador del aprendizaje, en el cual el que más importa es el estudiante y el "aprendizaje" entendido como el cambio de conducta o mecanización o repetición de actividades con el mínimo de errores. "En el proceso de orientación del aprendizaje, es de vital importancia conocer la estructura cognitiva del alumno; no sólo se trata de saber la cantidad de información que posee, sino cuales son los conceptos y proposiciones que maneja así como de su grado de estabilidad"(Ausubel, 1983) 


\section{Rev. SINAPSIS, Edición № 9, Vol. 2, Diciembre 2016}

Como refiere(Tunnermann, 2011) "La educadora ecuatoriana Rosa María Torres nos previene del error de exagerar el énfasis en los aprendizajes, hasta el extremo de desconocer la relación dialéctica que debe existir entre la enseñanza y el aprendizaje entre el profesor y el alumno. Al respecto, esta educadora nos dice, “CConcentrar la atención en el aprendizaje? Uno de los puntos nodales y promisorios de la "nueva visión” se refiere a “concentrar la atención en el aprendizaje”. La presente investigación busca incentivar a los docentes el mejorar su desarrollo profesional en el campo laboral y así elevar el nivel de aprendizaje de los estudiantes, su desarrollo de habilidades y destrezas, convirtiéndolos en seres humanos productivos.

Los recursos didácticos son un conjunto de elementos que facilitan la realización del proceso de enseñanza y aprendizaje, los cuales contribuyen a que los estudiantes logren el dominio de un conocimiento determinado, al proporcionarles experiencias sensoriales representativas de dicho conocimiento. Es cualquier material que, en un contexto educativo determinado, sea utilizado para facilitar el desarrollo de las actividades formativas. Los recursos educativos que se pueden utilizar en una situación de enseñanza y aprendizaje pueden ser o no medios didácticos.

Los medios didácticos pueden definirse como cualquier recurso que el profesor prevea emplear en el diseño o desarrollo del currículo para aproximar o facilitar los contenidos, mediar en las experiencias de aprendizaje, provocar encuentros o situaciones, desarrollar habilidades cognitivas, apoyar sus estrategias metodológicas, o facilitar o enriquecer la evaluación(Cacheiro, 2011)

Los Recursos Didácticos no son valiosos por sí mismos, su uso queda plenamente justificado cuando son integrados, de manera adecuada, al proceso educativo, el cual deberá ser compatible a su vez, con otros contextos más amplios, ya que despiertan un alto grado de interés en los estudiantes, proporcionando experiencia real que se obtienen mediante materiales y medios que estimulan la actividad por parte de los alumnos, desarrollando continuidad de pensamiento y contribuyendo al aumento de los significados.

El aprendizaje significativo, "facilita el aprendizaje del alumnado a través de la reflexión personal de sus experiencias -vividas dentro y fuera del aula-relativas a su proceso de aprendizaje, por lo que constituye una importante fuente de información sobre los procesos de autoevaluación, cumplimiento de los objetivos, funcionamiento y relaciones de la clase, aspectos socioculturales y afectivos que pueden ser 


\section{Rev. SINAPSIS, Edición № 9, Vol. 2, Diciembre 2016}

tanto o más relevantes que el aprendizaje, que el método, la presentación de los contenidos o los materiales"(Palomares, 2011)

Analizando los trabajos realizados en las Universidades de los países de Habla hispana referentes se encuentran tesis de investigación de pregrado y posgrado sobre la aplicación de estrategias metodológicas y aprendizaje significativo en diferentes áreas, muchas coinciden en que el material didáctico es básico para todos los alumnos; pues se motivan a aprender más y mejor siendo analíticos, razonadores de cosas de la vida, ya sean niños o jóvenes, ellos tienen derecho a recibir un buen material didáctico de educación.

Se representa "el aprendizaje como una construcción personal a partir de la reflexión, con lo cual se convierten en un medio útil para no olvidar lo que se aprende. El aprendizaje es significativo cuando los conceptos nuevos se van integrando en conceptos más amplios y así progresivamente"(Salinas, Darder, \& Benito, 2011)

La idea central de la teoría de Ausubel, es que de todos los factores que influyen en el aprendizaje, el más importante es lo que el alumno ya sabe. Es decir, aprendemos desde lo que ya sabemos. El aprendizaje significativo es aquel en el cual el alumno convierte el contenido de aprendizaje en significados para sí mismos. Esto quiere decir que el estudiante puede relacionar, de modo sustancial y no arbitrario, el contenido y la tarea del aprendizaje, con lo que él ya sabe.

Aprendizaje significativo es aprendizaje con comprensión, con significado, con capacidad de transferencia. Es el opuesto del aprendizaje mecánico. Si imaginamos que el aprendizaje se produce a lo largo de un continuo, aprendizaje mecánico estaría en un extremo y aprendizaje significativo en el otro. (Moreira, 2012)

La teoría del Aprendizaje Significativo tiene importante implicación psicológica y pedagógica. Considera que el aprendizaje se construye de manera evolutiva. Porque se ocupa de lo que ocurre en el aula, postula los principios programáticos para organizar la docencia y, en este sentido, adquiere un valor especial la necesidad de realizar un análisis conceptual del contenido.

Es indispensable identificar las características y competencias tanto del contexto como de los estudiantes, para obtener buenos resultados, buscando mejorar el nivel educativo de los alumnos; "reconocer aquellos 


\section{Rev. SINAPSIS, Edición № 9, Vol. 2, Diciembre 2016}

recursos y materiales educativos existentes y necesarios que repercuten en la calidad de la educación; y conocer su utilidad en el proceso de enseñanza aprendizaje. Por lo que es necesario dotar a los profesores de competencias que les permitan determinar criterios y factores para seleccionar estrategias didácticas y tecnologías educativas apropiadas para contribuir a este proceso de enseñanza aprendizaje"(Rivero, Marcela, \& Abrego, ecnologías educativas y estrategias didácticas: criterios de selección., 2013)

Cabe resaltar que el aprendizaje significativo se caracteriza por la interacción entre los conocimientos previos y los conocimientos nuevos planteados en un proceso de aprendizaje, adquiriendo significado para los nuevos conocimientos y los conocimientos previos adquieren mejor estabilidad.

(Metabase de Recursos Educativos, 2015)Refiere que "un aprendizaje significativo es necesario ya que es un aprendizaje con significado, comprensión, retención, capacidad de transferencia, que el objetivo que los profesores esperan como resultado de su acción docente”. Llegando así a los alumnos y evidenciando los resultados obtenidos, sería gratificante en nuestra experiencia como educador.

Los resultados de (Angelica, 2010) demuestran que las estrategias de aprendizaje, son concebidas como acciones generadas por quien aprende para aprender y controlar su aprendizaje, y además menciona reflexiones basadas en diferentes teorías entre la que podemos citar que "El aprendizaje significativo precisa la actividad por parte de quien aprende. Es una actividad de naturaleza interna, es una actividad cognitiva que puede estimularse mediante acción externa”.

La meta del aprendizaje significativo se basa en aprender a aprender, mediante las estrategias apropiadas que se encuentren al alcance del docente.

Diferentes investigaciones realizadas a nivel nacional e internacional referente al de tema de investigación, determinan que nuestro estudio logró obtener una etapa avanzada donde se pudo identificar la escaza aplicación de los recursos didácticos que conllevan a un aprendizaje significativo y productivo en las diferentes aulas de clase, logrando experimentar con nuevos recursos y de esta manera incentivando un aprendizaje integral. Es así que en la Universidad Central del Ecuador, con el tema de trabajo de investigación previo a la obtención del título de tercer nivel, refiere: "el aprendizaje conduce a cambios de larga duración en el comportamiento potencial.. Sin embargo, el solo potencial no es 


\section{Rev. SINAPSIS, Edición № 9, Vol. 2, Diciembre 2016}

suficiente: el aprendizaje necesita ser reforzado para que perdure. El aprendizaje es un proceso utilizado para la adaptación, la evolución, la supervivencia, los cambios en nuestro comportamiento, la adquisición de experiencia para la obtención de nueva información.(Caiza, 2012)

En varios países de América Latina donde se han ejecutado acciones tendientes a incorporar en sus sistemas educativos procesos innovadores, propuestos para obtener la eficiencia externa e interna en función de las necesidades y aspiraciones de la sociedad; sin embargo, en el ámbito educativo de la Educación Básica, se ha producido una corriente de opinión generalizada, que sostiene que este nivel educativo no responde a las necesidades y exigencias del medio.

De acuerdo con el requerimiento descrito, "el profesor en su actividad mediadora debe de hacer uso de estrategias, técnicas, métodos y recursos que al ser utilizados en el proceso educativo, puede generar o promover un aprendizaje significativo"(Vera \& Luis, 2011). Al respecto, la Organización de la Naciones Unidas para la Educación, Ciencia y Cultura. Recomienda dotar al alumno de la posibilidad de desarrollar habilidades como la comprensión verbal, numérica, visualización espacial, retención de imágenes, palabras y razonamiento, entre otras.

Como enfatizan(Rivero, Gómez, \& Abrego, Tecnologías Educactivas y Estrategias Didácticas: Criterios de Seleccción., 2013) “La Temática de los recursos didácticos están revolucionando la práctica educativa, exigiendo cada vez más que el docente identifique factores y establezca criterios para incorporar aquellas tecnologías educativas y estrategias didácticas de calidad en su planeación y organización educativa, haciendo así más fácil llegar a los estudiantes llamando su atención e interés”. Es sustancial incentivar al docente a la innovación para así mejorar el nivel académico de los alumnos.

De igual manera refiere (Del Moral \& Villalustre, 2012) "la progresiva y creciente planificación de la enseñanza en términos de competencias ha supuesto un cambio significativo en la práctica educativa del profesorado que requiere de una capacitación constante - profesional específica para ayudar a identificar fortalezas y debilidades y acompañar al estudiante en su proceso formativo", encaminando a la mejora continua del proceso enseñanza Aprendizaje y mejorando así el panorama del aprendizaje. 


\section{Rev. SINAPSIS, Edición № 9, Vol. 2, Diciembre 2016}

Es importante analizar que actualmente la tecnología debe utilizarse como herramienta del recurso didáctico como refiere (Romero \& Quesada, 2014)“la aplicación de lasTIC a la enseñanza de las ciencias llega al estudiante de una manera más fácil adquiriendo conocimiento. El valor formativo de dichas aplicaciones se discute desde los actuales conocimientos acerca de cómo los individuos aprenden, mostrando el potencial de estos recursos para superar los obstáculos específicos asociados al aprendizaje efectivo"

Por esa razón, una de las mayores dificultades que confrontan los sistemas educativos latinoamericanos, es el bajo rendimiento académico de los estudiantes, definido éste, como el nivel de aprendizaje alcanzado por los alumnos. Esta situación, deriva de la pobreza cultural, programas ambiguos, objetivos superficiales y de los métodos y estrategias inoperantes tradicionales que aplican algunos docentes.

\section{Materiales y Métodos.}

Para recopilar la información de la presente investigación, se hizo uso del método bibliográficodocumental, donde se obtuvo documentos alusivos al tema que son de gran utilidad para la información, así como: folletos, libros y otros materiales que sirvieron de fundamento para el desarrollo de la tesis, para realizar comparaciones profundizar, y deducir diferentes enfoques de teorías, conceptualizaciones y el criterio de varios autores.

Para optimizar y desarrollar el trabajo investigativo de la tesis, se utilizó la investigación de campo que se realizó en el mismo lugar del fenómeno de estudio, donde se encuentran los directivos, docentes y estudiantes del Colegio Nacional "Alejo Lascano". Aquí se toma contacto directo con la realidad y se obtuvo la información de acuerdo a los objetivos planteados.

El nivel de investigación fue descriptivo-propositivo ya que por medio de los resultados se recopilo científicamente los diversos métodos de aprendizajes que sirvieron de base para elaborar la propuesta y potenciar el rendimiento académico del Sistema Educativo.

La técnica utilizada fue la encuesta dirigida a los docentes y alumnos de bachillerato, se recopiló la información para caracterizar la investigación de los métodos de aprendizaje y al desarrollo de la credibilidad en los estudiantes del Colegio Nacional "Alejo Lascano" a su vez con los resultados obtenidos se elaboró la propuesta. 


\section{Rev. SINAPSIS, Edición № 9, Vol. 2, Diciembre 2016}

En el trabajo investigativo se aplicó los instrumentos de medición y resultantes del producto de las matrices de la operacionalización de las variables, las mismas que tienen relación con los objetivos planteados.

La Entrevista se la aplicó a los docentes, directivos de la institución, se realizó con el fin de obtener información".

\section{Resultados}

Durante el periodo de la investigación se pudo identificar mediante la aplicación de la encuesta dirigida a los docentes, que estrategias metodológicas los docentes utilizan, relacionándolo con el nivel de interés en el aprendizaje de los estudiantes. La importancia que tiene el tomar en cuenta la participación de los alumnos en las clases. Establecer la formación didáctica de los docentes para implementar estrategias innovadoras de enseñanza a los estudiantes.

TABLA \# 1

\section{¿Qué medios didácticos utiliza para el desarrollo de la clase?}

\begin{tabular}{lcc}
\multicolumn{1}{c}{ ALTERNATIVAS } & f & \% \\
\hline Texto, Pizarrón y Tiza & 45 & 73 \\
\hline Material Audiovisual Interactivo & 5 & 8 \\
\hline Recursos del Medio Ambiente & 7 & 11 \\
\hline Otros & 5 & 8 \\
\hline Ninguna & 0 & 0 \\
\hline TOTAL & $\mathbf{6 2}$ & $\mathbf{1 0 0}$ \\
\hline
\end{tabular}

FUENTE: Encuesta a Docentes del Colegio "Nacional Alejo Lascano"

ELABORACIÓN: Elvira Geoconda Villacreses Veliz

La tabla representa a cuarenta y cinco docentes, el 73\%, utilizan medios didácticos como Texto, Pizarrón y Tiza, cinco docentes, el $8 \%$ indicaron que utilizan materiales audiovisuales interactivos; siete docentes, $11 \%$ utilizan los recursos del medio ambiente, cinco, el $8 \%$ dijeron otros, y por la alternativa ninguno no hubo respuesta. 


\section{Rev. SINAPSIS, Edición № 9, Vol. 2, Diciembre 2016}

En el desarrollo de la investigación fue primordial identificar lo que el alumno ve que no es capaz de seguir estudiando, ya sea porque el trabajo es demasiado y no sabe cómo explotar sus habilidades de estudio o porque su rendimiento académico es insuficiente provocando desanimo a la hora de estudiar. Por tal razón se considera importante que el docente conozca sobre los medios didácticos apropiados al momento de llevar a cabo el proceso de enseñanza-aprendizaje.

\section{TABLA \# 2}

3. ¿Qué importancia tiene el tomar en cuenta la participación de los alumnos en las clases?

\begin{tabular}{lccc} 
& ALTERNATIVAS & f & \% \\
\hline Mucha & 62 & 100 \\
\hline Poca & 0 & 0 \\
\hline Ninguna & 0 & 0 \\
\hline TOTAL & $\mathbf{6 2}$ & $\mathbf{1 0 0 \%}$ \\
\hline
\end{tabular}

FUENTE: Encuesta a Docentes del Colegio "Nacional Alejo Lascano"

ELABORACIÓN:Adela del Jesús Lucio Pillasagua y Elvira Geoconda Villa creses Veliz

Una vez realizada la encuesta a docentes, en lo que respecta a esta pregunta se obtuvieron los siguientes resultados: 62 docentes el 100\% contesto que es muy importante la participación de los estudiantes dentro del aula de clases para obtener un aprendizaje significativo, y por la alternativa Poca y Ninguno no hubo respuestas.

La participación de los estudiantes dentro del aula de clases es un compromiso activo para prestar atención a su inserción dándoles la oportunidad de demostrar el interés por colaborar en el proceso de aprendizaje, es importante que se relacionen las tareas asignadas con sus metas, lo que ayudara a motivar al estudiante para obtener un alto rendimiento, a superar problemas conductuales y a mantener buenas relaciones interpersonales dentro de la Institución Educativa por lo consiguiente se debe involucrar a los estudiantes en todo el proceso de aprendizaje como parte de su formación, el docente es el encargado de motivar al alumno para que obtenga dentro del salón de clases una mejor comprensión y un rendimiento académico óptimo. 
Rev. SINAPSIS, Edición № 9, Vol. 2, Diciembre 2016

ISSN 1390 - 9770

TABLA \# 3

\section{4.- ¿Qué actividad realiza para dinamizar sus clases?}

\begin{tabular}{lcc}
\multicolumn{1}{c}{ ALTERNATIVAS } & f & \% \\
\hline Forma equipos de trabajo & 42 & 68 \\
\hline Rompe la rutina con dinámicos de animación & 17 & 27 \\
\hline Realiza ejercicios de aplicación & 1 & 1 \\
\hline Utiliza otro recurso & 2 & 4 \\
\hline TOTAL & $\mathbf{6 2}$ & $\mathbf{1 0 0}$ \\
\hline
\end{tabular}

FUENTE: Encuesta a Docentes del Colegio "Nacional Alejo Lascano"

ELABORACIÓN: Adela del Jesús Lucio Pillasagua y Elvira Geoconda Villa creses Veliz

Con respecto a la pregunta, 31 Docentes que representan 68\% Forma Equipos de Trabajo dentro del aula de clases, 16 docentes que representan el 27\% Rompe la rutina con dinámicos de animación, 1 docente que representan $1 \%$ realiza ejercicios de aplicación y 2 docentes que representa el $4 \%$ utiliza otro recurso.

La capacitación del docente debe estar orientada no solo a incrementar o actualizar los conocimientos del docente, sino a encontrar nuevas maneras de utilizarlos en el mundo de hoy.

Es decir que los docentes necesitan aplicar métodos y técnicas que permitan establecer contacto con el estudiante descubriendo conocimientos para trasmitir el contenido de la materia. La capacidad para resolver problemas es la meta principal de la educación, cada joven debería ser un pensador creativo y crítico originando motivación y confianza en sí mismo.

\section{ENCUESTA A ESTUDIANTE DEL COLEGIO NACIONAL ALEJO LASCANO DEL CANTÓN JIPIJAPA.}

Durante el periodo de la investigación se pudo identificar mediante la aplicación de la encuesta dirigida a los estudiantes, que recursos didácticos y estrategias utiliza el profesor en el desarrollo de la clase.

\section{TABLA \# 4}


Rev. SINAPSIS, Edición № 9, Vol. 2, Diciembre 2016

ISSN 1390 - 9770

4.- ¿Qué recursos didácticos y estrategias utiliza el profesor en el desarrollo de la clase?

\begin{tabular}{lcc}
\hline \multicolumn{1}{c}{ ALTERNATIVAS } & F & \% \\
\hline Tiza líquida- pizarrón & 130 & 52 \\
\hline Proyector & 4 & 2 \\
\hline Paleógrafo & 66 & 26 \\
\hline Folletos & 17 & 7 \\
\hline Debate & 29 & 11 \\
\hline Videos & 4 & 2 \\
\hline Conferencias pregrabadas & 0 & 0 \\
\hline TOTAL & $\mathbf{2 5 0}$ & $\mathbf{1 0 0}$ \\
\hline
\end{tabular}

FUENTE: Encuesta a Docentes del Colegio "Nacional Alejo Lascano"

ELABORACIÓN: Adela del Jesús Lucio Pillasagua y Elvira Geoconda Villa creses Veliz

Los resultados de la tabulación a esta interrogante, son muy importantes ya que registra la realidad de los medios con los que cuenta el docente en el momento de impartir sus clases. El 52\% menciona que el recursos didáctico más utilizado es la pizarra y la tiza liquida, el paleógrafo en un26\%, el proyector en un $2 \%$, los folletos en un 7\%, el debate en un $11 \%$ y los videos en $2 \%$. Estos resultados reflejan que los docentes de la Institución educativa Alejo Lascano, no tienen a la mano los recursos didácticos innovadores, ya sea porque la institución no los ha podido proveer, o porque el docente no se ha capacitado adecuadamente según las necesidad del plantel educativo.

La calidad de estos procesos depende de la interacción entre profesores y alumnos en el aula, a las ayudas que los profesores ofrecen en esos procesos y al grado en que estas ayudas se ajusten a los recursos cognitivos, motivacionales, emotivos y relacionales y que ponen en marcha para aprender.

A veces los estudiantes no aprenden porque no están motivados y por ello no estudian, pero otras veces no están motivadas precisamente porque no aprenden, ya que utilizan estrategias de aprendizaje inadecuadas que les impiden experimentar la sensación de "saber que se sabe aprender"

\section{TABLA \# 5}


Rev. SINAPSIS, Edición № 9, Vol. 2, Diciembre 2016

7.- ¿Los profesores aplican métodos que generan aprendizajes significativos?

\begin{tabular}{ccc}
\hline ALTERNATIVAS & f & \% \\
\hline Siempre & 80 & 32 \\
\hline Rara vez & 128 & 51 \\
\hline A veces & 42 & 17 \\
\hline Nunca & 0 & 0 \\
\hline TOTAL & $\mathbf{2 5 0}$ & $\mathbf{1 0 0}$ \\
\hline
\end{tabular}

FUENTE: Encuesta a Docentes del Colegio "Nacional Alejo Lascano"

ELABORACIÓN: Adela del Jesús Lucio Pillasagua y Elvira Geoconda Villacreses Veliz

Los resultados obtenidos en el cuadro anterior se presentan de la siguiente manera: el $32 \%$ de los estudiantes manifiestan que los docentes aplican siempre el aprendizaje significativo en el momento de impartir sus clases, el 51\% manifiestan que los docentes a veces aplican en sus horas clase el aprendizaje significativo, el $17 \%$ establecen que los docentes rara vez aplican aprendizajes significativo. De esta manera podemos analizar que la metodología del docentes aplicando el aprendizaje significativo es en un $32 \%$ que nos da la pauta que el $68 \%$ de los docentes, no imparten en sus horas clase aprendizajes significativo, y cabe recalcar que este es de suma importancia en el desarrollo del ser humano porque todo lo aprendido en el salón de clase se lo lleva a la práctica en la vida diaria y este aprendizaje lo llevará toda la vida el alumno.

La información se convierte en un recurso útil y a la disposición de la persona, en el momento necesario. Experimentación directa sobre la realidad, aplicación práctica de los conocimientos y su transferencia a diversas situaciones. Aprendizaje por penetración comprensiva.

Se puede evidenciar en un alto porcentaje que la enseñanza debe proporcionar entornos de aprendizaje ricos en recursos educativos, en los que los estudiantes puedan desarrollar actividades que les permitan descubrir el conocimiento, aplicarlo en situaciones prácticas y desarrollar todas sus capacidades (experimentación, descubrimiento, creatividad, iniciativa.).

TABLA \# 6 
Rev. SINAPSIS, Edición № 9, Vol. 2, Diciembre 2016

6.- ¿Existe creatividad durante el desarrollo de sus clases?

\begin{tabular}{ccc}
\hline ALTERNATIVAS & f & $\boldsymbol{\%}$ \\
\hline Siempre & 83 & 33 \\
\hline Rara vez & 125 & 50 \\
\hline A veces & 42 & 17 \\
\hline Nunca & 0 & 0 \\
\hline TOTAL & $\mathbf{2 5 0}$ & $\mathbf{1 0 0}$ \\
\hline
\end{tabular}

FUENTE: Encuesta a Docentes del Colegio "Nacional Alejo Lascano"

ELABORACIÓN: Adela del Jesús Lucio Pillasagua y Elvira Geoconda Villacreses Veliz

La tabulación de este gráfico arroja los siguientes resultados: el 33\% de los estudiantes mencionan que siempre existe creatividad del docente en el desarrollo de sus clases, el 50\% menciona que rara vez se aplica la creatividad en sus clases, el $17 \%$ responde que a veces el docente imparte las clases con creatividad, y el $0 \%$ indica que nunca. Estos valores reflejan la necesidad que tienen los docentes de actualización en el campo de la didáctica-creatividad y pedagogía, ya que los alumnos de hoy, son más exigentes, porque la sociedad con los avances tecnológicos y científicos se los permite, esta lleva al estudiantado a estar acorde a la información que tienen a la mano y de la misma manera el docente tiene la obligación de prepararse y buscar los medios adecuados para que la clase se desarrolle con agrado y sea receptiva para los educandos

\section{Discusión}

Es relevante en el accionar del Docente tomar muy en cuenta el uso adecuado del material didáctico y las estrategias a utilizar al momento de impartir conocimientos de su cátedra, ya que de las estrategias que este utilice depende mucho el éxito de su clase para evitar los altísimos índices de fracaso escolar. Se establece esta importancia en los diferentes estudios realizados a nivel nacional e internacional, recalcando que esta investigación tiene una aplicación experimental en el campo de investigación que arrojo buenos resultados, demostrando que si se aplican recursos didácticos orientados a un aprendizaje significativo, estamos formando seres productivos para la sociedad. 


\section{Rev. SINAPSIS, Edición № 9, Vol. 2, Diciembre 2016}

En muchas ocasiones el fallo está en una carencia de hábitos y técnicas de estudio en las instituciones educativas y las evidencias muestran que la mayor parte de los estudiantes no aprenden las habilidades de estudio si no reciben enseñanza explicita sobre su uso, esto quiere decir que el alumnado no tiene la capacidad de usar una técnica de estudio en forma apropiada y eficiente.

Dentro del interés por la investigación sobre el aprendizaje de los estudiantes, uno de los aspectos más importantes a tratar es la utilización de técnicas de estudio, habilidades de estudio y estrategias de aprendizaje.

El alumno ve que no es capaz de seguir estudiando, ya sea porque el trabajo es demasiado y no sabe cómo explotar sus habilidades de estudio o porque su rendimiento académico es insuficiente provocando desanimo a la hora de estudiar. Por tal razón se considera importante que el docente conozca sobre los medios didácticos apropiados al momento de llevar a cabo el proceso de enseñanza-aprendizaje.

\section{Conclusiones}

a) Se establece el escaso apoyo de las autoridades gubernamentales ya que la cantidad de estudiantes y necesidades que presentan las Instituciones Educativas de la zona Sur son muy grandes y el centro educativo necesita dotarse de medios y recursos didácticos de tecnología y así mismo del espacio físico adecuado para su desarrollo, encaminado al buen rendimiento escolar que incentive al docente en el desarrollo de sus clases y motivar al estudiante para despertar el interés de estos y obtener mejores resultados.

b) Se determina que las y los estudiantes no reciben suficiente motivación en las clases impartidas por la escasa aplicación de recursos didácticos y de estrategias motivadoras que los docentes deben agregarles como componente para aumentar el interés en los contenidos del proceso de enseñanza, derivándose esta de la incidencia negativa del modelo tradicional empleado y que repercute en el rendimiento escolar.

c) Se percibe la escasa motivación de las y los estudiantes como el resultado del desinterés de los docentes por la aplicación de métodos y estrategias que estimulen a la participación activa en el proceso de enseñanza aprendizaje, reflejado en la falta de compromiso con las tareas diarias en los dicentes. 


\section{Rev. SINAPSIS, Edición № 9, Vol. 2, Diciembre 2016}

d) Los estudiantes evidenciaron el escaso compromiso de los docentes por mejorar su desarrollo profesional al aplicar metodologías que producen el desmejoramiento del rendimiento que afecta directamente al estudiante ya que los conocimientos impartidos por los docentes no son recibidos con agrado por el dicente, es decir se trabaja sin tener buenos resultados.

\section{Bibliografía}

Angelica, V. L. (2010). Estrategias de aprendizaje. Gondola, 4.

Antonio, M. M. (2010). ¿Al Final, Qué es prendizaje Significativo? Qurriculum, 2.

Aparici, R. (01 de Mayo de 2011). Organizaciones de los Estados Americanos. Obtenido de La Educación: http://www.educoas.org/portal/laeducacion2010

Ausubel, D. (01 de Agosto de 1983). Psicología Educativa y el labor docente. En D. D. Ausubel, \& O. L. Castiblanco abril (Ed.), Teoria del Aprendizaje Significativo (Vol. 5, pág. 1). Mexico: Index. doi:Pp27-37

Cacheiro, M. (01 de Julio de 2011). Revistas de Medios y Educación. Obtenido de Revistas de Medios y Educación: http://acdc.sav.us.es/pixelbit/images/stories/p39/06.pdf

Caiza, M. (9 de Junio de 2012). Repositorio digital UCE. Obtenido de Incidencia de la atención dispersa en el aprendizaje: http://www.dspace.uce.edu.ec/handle/25000/253

Del Moral, M. E., \& Villalustre, L. (2012). Didáctica Universitaria en la era 2.0: Competencias Docentes en Campos Virtuales. Revista de Universidad y Sociedad del Conocimiento volumen 9, 4.

Metabase de Recursos Educativos. (2015). Teorías psicológicas del aprendizaje. Mexico: e-UAEM.

Moreira, M. (2012). La Teoría del Aprendizaje Significativo Crítico: un r3eferente para organizar la enseñanza contemporanea. Revista Iberoamericana de Educación, 4.

Palomares, A. (2011). El modelo docente universitario y el uso de nuevas metodologías en la enseñanza, aprendizaje y evaluación. Revista de Educación, 3. Obtenido de Revista de Educación: http://www.revistaeducacion.educacion.es

Rivero, I., Gómez, M., \& Abrego, R. F. (2013). Tecnologías Educactivas y Estrategias Didácticas: Criterios de Seleccción. Educación y Tecnología, N³, 191.

Rivero, I., Marcela, G., \& Abrego, R. (2013). ecnologías educativas y estrategias didácticas: criterios de selección. Revista Educación y Tecnología, 3.

Romero, M., \& Quesada, A. (2014). Nueva tecnología y el Aprendizaje Significativo de la Ciencia. Dipósit Digital de Documents de la UAB, 2. 
Rev. SINAPSIS, Edición № 9, Vol. 2, Diciembre 2016

ISSN $1390-9770$

Salinas, J., Darder, A., \& Benito, B. d. (2011). Los mapas conceptuales como organizadores del proceso enseñanza aprendizaje: los itinerarios de aprendizaje. Investigación Novación Educativa i socioeducativa., 5 .

Tunnermann, C. (2011). El Constructivismo y el Aprendizaje de los Estudiantes. Red de Revistas Científicas de América Latina, el Caribe, España y Portugal, 3 a 13.

Vera, A., \& Luis, V. (2011). Estrategias utilizadas por los docentes para promover el aprendizaje de la biología. Dialnet, 3. 